\title{
UNA REVISIÓN AL EJERCICIO DEL PODER Y EL CAMBIO ORGANIZACIONAL EN LA EMPRESA FAMILIAR
}

\author{
Autores: Aylin Patricia Pertuz Martínez (1) \\ Álvaro Enrique Santamaría Escobar (2) \\ Wilson Guzmán Cadrazco Parra (3)
}

\section{RESUMEN}

$\mathrm{E}^{\prime}$ estudio del poder tiene una historia reciente en la literatura organizacional. El poder se encuentra presente en la vida de las empresas, haciendo parte de la vida política de la misma. El cambio organizacional es uno de los procesos donde el poder se manifiesta. En las empresas familiares tiene dimensiones particulares en la sucesión y el relevo generacional. El objetivo de esta revisión es señalar las formas de intervención del poder en el cambio organizacional, en la vida de la empresa familiar y cómo actúa en los procesos de sucesión y cambio o relevo generacional. Se concluye que el ejercicio del poder toma fuerza en el cambio organizacional y muestra su complejidad en las empresas familiares; en éstas, los juegos de poder tienden a ser una traba en los procesos de sucesión y relevo generacional; el éxito o no de estos procesos podrá depender del modo en cómo se desarrolle el ejercicio del poder.

Palabras claves: poder, cambio organizacional, empresa familiar, relevo generacional

\begin{abstract}
$\mathrm{T}$ he organizational change is one of the processes where power struggle emerges. Family businesses have certain particular features concerning the succession and hand over processes. This research has as main purpose to review how the hand over and succession processes affect the organizational and the organizational practices in family businesses. This study concludes that the exercise of power affect organizational change making more complex the relationships in their heirs. The success or failure of these processes lies on the way they exercise power in such situations.
\end{abstract}

Keywords: power, organizational change, family business, generational change.

(1) Administradora de Empresas, Magistra en Administración de Empresas, Especialista en Gestión Pública, Doctoranda en Ciencias Sociales Mención Gerencia; Docente de planta Universidad de Sucre; aylin.pertuz@unisucre.edu.co

(2) Ingeniero Industrial, Especialista en Gerencia de la Producción y la Calidad, Magister en Administración de Empresas, Magister en Educación, Doctorando en Ciencias Sociales Mención Gerencia. Docente de planta de la Universidad de Sucre. Grupo de investigación en Gestión de la Producción y la Calidad Organizacional.

(3) Administrador de Empresas, Especialista en Alta Gerencia, Magister en Administración de Empresas, Doctorando en Administración de Empresas. Docente de planta de la Universidad de Sucre. Grupo de investigación en Gestión de la Producción y la Calidad Organizacional 


\section{INTRODUCCIÓN}

$\mathrm{E}$ 1 presente artículo tiene como objetivo, señalar las formas de intervención del poder en el cambio organizacional, en la vida de la empresa familiar y cómo actúa en los procesos de sucesión y relevo generacional en este tipo de empresas. Para ello, se llevó a cabo una revisión de la literatura, en la cual se aborda esta temática en forma directa o relacionada. La revisión comprendió artículos de revistas arbitradas, tesis de maestría, tesis doctorales y textos en el área organizacional. Se hizo uso de la base de datos Proquest y Scopus, así como la base de las revistas electrónicas Orbis y Negotium. Es analítico interpretativo.

Presenta cuatro secciones: en la primera, se expone la conceptualización del poder desde la filosofía y el campo organizacional; la segunda sección, trata la interrelación entre el poder y el cambio organizacional; en la tercera, se une el poder a la naturaleza de la empresa familiar, y, en la cuarta sección, se exponen los procesos de sucesión y cambio generacional en la empresa familiar, tomando como eje el poder. Finaliza con las conclusiones.

\section{EL EJERCICIO DEL PODER}

El tema del poder tiene en Michel Foucault a uno de los estudiosos más reconocidos. El concepto que el autor arroja acerca del poder ha tenido varias modificaciones en su producción. Inicialmente, en los años sesenta el poder lo define como represivo, negativo, limitador de la libertad; perspectiva que es modificada en los setenta, en donde el poder se asume como relaciones de poder (Rodríguez, 2000:2).
De esta manera, para Foucault:

"El poder sólo existe como acción que actúa sobre otra $u$ otras acciones que se han dado o que están en el límite de un poder ser. Su ejercicio es guiar la posibilidad de la conducta; el problema del poder es un problema de gobierno, de política: su fin está en obtener, mediante una acción calculada, una determinada acción esperada de un sujeto individual o colectivo en un campo de acción con diversas posibilidades y formas de conducirse".

No existe confrontación entre poder y libertad, más bien cada uno es la condición de existencia del otro. En las relaciones de poder unos sujetos tienen la intencionalidad de determinar la conducta de otros, pero no en forma imperativa por la coerción, pues de ser así, existiría un sometimiento por la fuerza y no una relación de poder. (Rodríguez, 2000)

Foucault plantea que el poder no es una propiedad, no es una prohibición que un sujeto imponga sobre otro; el poder atraviesa a todos los sujetos, incluyendo a las personas que se le intentan resistir. El poder es positivo, si fuera negativo sería sólo represión. Sin embargo, la tendencia de la sociedad occidental es la de presentar el poder como negativo; esto se entiende desde "el ejercicio mismo del poder cuya eficacia está dada en relación con su capacidad de esconder sus mecanismos". El poder es omnipresente, se produce en cada instante y en cada parte interrelacionado con todo, "el poder no engloba, pero viene de todas partes" (Rodríguez, 2000).

En el campo organizacional, el estudio del poder es reciente, destacándose autores como Pfeffer (1981), Minztberg (1983), Morgan (1986), Daudi (1986), Robins y Coulter (2005), Zaleznick y Kets de Vries (1975), Hall (1996), entre otros (De la Garza, Melchor y Jiménez (2008). 
Para Morgan (1998), el poder es uno de los elementos de las organizaciones como sistemas políticos. Según el autor, "el poder es el medio a través del cual los conflictos de intereses son resueltos en última instancia. En el poder influye quién lo ejerce, qué cuándo y cómo". De acuerdo con Dahl, el poder "implica una habilidad de hacer que otra persona haga algo que de otra manera no habría hecho". En su exploración del poder, Morgan expone catorce fuentes de poder, que van desde la autoridad formal, pasando por el control de los procesos de decisión, control de los recursos escasos, hasta el poder que ya se tiene.

\section{PODER Y CAMBIO ORGANIZACIONAL}

El cambio es una constante hoy en día. Los programas de cambio son una necesidad, motivada por las transformaciones de las relaciones y del tiempo en que están inmersas las organizaciones actuales. Pero, no todos los cambios son iguales. De acuerdo con la literatura organizacional, es necesario distinguir los cambios rutinarios de los cambios planificados. Estos últimos, pretenden preparar a la organización, o, parte de ella, para adaptarse a los cambios significativos del entorno (Stoner, Freeman y Gilbert, 1996).

Los cambios, en términos de la transición pueden ser de tres formas: los cambios evolutivos, que son lentos, relacionados con el crecimiento natural y consolidación de la organización; los cambios revolucionarios, que se presentan de forma inesperada; y los cambios sistemáticos, que se dan de forma planeada, constante pero no tan rápidos, siendo el más apropiado en cuanto a las resistencias que pueden darse en el proceso (Quirant y Ortega (2006), citado en Osorio y Ravelo, 2011).

Para el proceso de cambio, es reconocido el modelo Lewin (1950), perfeccionado más tarde por
Schein en tres pasos: descongelar, cambiar y recongelar (Stoner, et al, 1996). Gibson , Ivancevich, Donnelly y Konopaske (2006), por su parte, presenta un modelo de siete pasos desde la identificación de fuerzas para el cambio, pasando por el diagnóstico del problema, la implementación, hasta la evaluación del método.

Los procesos de cambio organizacional, se constituyen en uno de los aspectos en los cuales juega el poder, sin importar si son cambios originados por factores de contexto externo, tal como presiones de los clientes, llegada de nuevos competidores, cierres de mercados por factores políticos; o internos, como reorganizaciones configurativas, implementación de nuevos sistemas, relevos generacionales, entre otros.

Según Peffer, el poder cobra importancia en actividades como restructuraciones, cambios de personal y asignación de recursos, de esta manera, el uso del poder se evidencia en la toma de decisiones cuando se ven las preferencias hacia determinados sectores o personas que no cumplen con los requerimientos adecuados (Chaux, 2006)

De acuerdo con Lewin (1951), en los procesos de cambio organizacional es importante reconocer que existen fuerzas impulsoras y otras que son restrictivas. Una fuerza impulsora puede ser la oportunidad de una tecnología nueva, y una fuerza restrictiva, el temor al cambio. El aumento de las fuerzas impulsoras puede hacer mejorar los resultados, pero también puede hacer crecer las fuerzas restrictivas (Stoner et al, 1996). En estas últimas, existen dos fuentes de resistencia: las fuentes individuales y las organizacionales. En las organizacionales, el cambio se considera "una amenaza a las relaciones establecidas de poder". Cambiar la configuración, por ejemplo, es una amenaza para quienes detentan el poder (Acosta, 2002). 
En la dinámica de un cambio existen tres focos: la percepción, el poder y el realismo. La percepción es una "impresión materializada" en los sentidos ante el entorno exterior; el poder es el insumo indispensable en la "ruptura" que, en unión con la percepción y el realismo permiten dinamizar el proceso; el realismo ayuda a ubicarse en la situación actual y mirarse hacia un futuro o el escenario más probable, teniendo en cuenta escenarios que pueden ser alternativos (López, Restrepo y López, 201

Ahora bien, el tiempo para la puesta en marcha de una estrategia de cambio depende de diversas variables, puede ser rápido o lento, como se anotaba anteriormente. Dentro de esas variables se encuentra el uso del poder, el cual se vincula con el "enfoque de resistencia, es decir, lo que se pretenda con ella, vencerla o minimizarla". $\mathrm{Si}$ el cambio es "revolucionario", lo que se aspira hacer es desaparecerla por completo; si el cambio es "evolutivo", la labor es de convencimiento en forma paulatina para derribar barreras (García, 2002).

En la resistencia al cambio, el poder adquiere relevancia debido a que la resistencia no es tanto hacia el cambio sino a la posibilidad de perder la posición o el acceso a los recursos (Font, Gudiño, Sánchez, 2001). En estudio realizado en empresas de confecciones de una ciudad colombiana, las relaciones de poder se constituyen en la variable que más presenta reticencia al cambio. Las categorías más influyentes son: la percepción de que los cambios sólo pueden ser propuestos y puestos en marcha por los directivos, $\mathrm{y}$, el estilo directivo que fomenta muy poco la autonomía del trabajador, lo cual hace que el personal se apegue a lo tradicional y no le interese lo nuevo o diferente (Montealegre y Calderón, 2007).

En lo que respecta al manejo del cambio planificado, se pueden utilizar modelos alternativos, tales como: el cambio mediante el poder, a través de la razón y por medio de la reeducación. En el primer caso, el uso del poder para obtener un cambio implica la coerción. Los administradores que detentan el poder lo usarán para obligar al personal a cambiar en "la dirección que se desea". Las recompensas y castigos serán los medios para ejercer el poder. Este manejo del poder involucra un estilo de liderazgo autocrático por parte de la gerencia (Gibson et al, 2006)

Por otra parte, teniendo en cuenta que las organizaciones son sistemas abiertos que se enfrentan y responden a los cambios que surgen en el entorno, $\mathrm{y}$, por lo tanto, las diferentes partes del entorno generan cambios al interior de la organización, se hace necesario, una diferenciación en lo estructural para hacerle frente a los cambios específicos. En este caso, una fuente de poder se relaciona con la incertidumbre y, por lo tanto, los subsistemas más poderosos serán los que se relacionen con esta incertidumbre y logren reducirla (Rodríguez, 1992)

Al nivel interno, los cambios también tienen profunda conexión con las coaliciones organizacionales. De esta manera, cuando la coalición dominante de una organización desea mantenerse en el poder, tratará de mantener las "características nucleares", en forma tal que el cambio genere un costo demasiado alto, haciendo que la organización se entregue a la "inercia estructural" (Caroll y Hannan, 1995) y evite el cambio, perpetuándose, entonces, la coalición dominante y las fuentes de poder al interior de la organización (Sanabria, Trujillo y Guzmán, 2008).

Asimismo, el poder se relaciona con otros elementos organizacionales, siendo dos de ellos, la comunicación y la estrategia. En la comunicación, puesto que los distintos medios son estrategias de poder sustentada en elementos que los grupos de la organización valoran, es decir, su cultura. En estudio empírico se demuestra que el uso de circulares 
abiertas incide en el cambio de una organización (Arras, Jaquez y Fierro 2008). En un proceso de cambio estratégico, en la fase de alineación, es necesario estar atentos a los factores de poder y de conflicto que están presentes en los actores y grupos de interés (Savarse, 2013).

\section{LA EMPRESA FAMILIAR Y EL PODER}

A nivel mundial, las empresas familiares son generadoras de riqueza y empleo o, en otras palabras, bienestar económico y social (Díaz, 2011; Rodríguez, Pico y Méndez, 2013). Aunque la empresa familiar suele identificarse con empresas pequeñas, también es un tipo de estructura presente en las grandes empresas (Tapies, 2011).

La literatura de la empresa familiar es copiosa con autores como: Litz (1995), Chua et al (1999), Shanker y Astrachan (1996), Neubaker y Lank (1999), resaltados por Gimeno (2004); Poulain (2006), destacado por Rodríguez, et al (2013:782); Gasson et al (1998), Daly y Dollinger (1992), señalados por García y Sánchez (2009).

En el análisis de la empresa familiar, son reconocidos los modelos conceptuales de los tres círculos (Tagiuri y Davis, 1982); el evolutivo tridimensional de Gersik et al (1997) (Bolaños, Pantoja, Jiménez, 2009); los cinco círculos de Amat y Salas (2004); y, el modelo basado en herramientas de identificación de la firma Consultoría Empresarial y Jurídica Suárez \& Asociados y Puente \& Asociados (Zuluaga, 2010).

A pesar de la amplia bibliografía, aún no existe una definición unánime del concepto de empresa familiar. Los primeros aportes provienen de autores como Christensen (1953), Donnelley (1964) y Levinson (1971). Posteriormente surgen publicaciones como, por ejemplo, las de Handler (1989), Whest- head y Coeling (1998), Shanker y Astracham (1996). El impulso en las investigaciones de los años recientes, tiene como base la magnitud de estas empresas en los PIB de los distintos países. (Vallejo, 2005).

El espectro de definiciones comprende las de carácter unidimensional, centradas en la propiedad, la dirección, la participación de la familia, el relevo generacional, y, las de carácter multidimensional, centradas en la propiedad y la dirección, así como las definiciones centradas en la propiedad, la dirección y una tercera dimensión adicional. (Vallejo, 2005).

En algunas instancias, se define a la empresa familiar como aquella en que "la propiedad o el poder de decisión pertenecen en todo o en parte a un grupo de personas que son parientes entre sí" (Egea, 2007). Por su parte, Vallejo (2005), la define de la siguiente forma:

La empresa familiar es aquella en que los miembros de una misma familia tienen una participación suficiente en el capital para dominar las decisiones propias del órgano de representación de propietarios, tenga éste carácter formal o legal o por el contrario sea de naturaleza informal, y en la que, además existe el deseo o la voluntad de continuidad del negocio en manos de la siguiente generación familiar.

Diversos aspectos han sido investigados por los académicos y consultores de las empresas de familia. Temas como, innovación, estrategia, cultura organizacional, emprendimiento, comunicación, gestión humana, finanzas, empresarismo, responsabilidad social, valores, poder, entre otros, dominan las esferas de investigación.

La empresa familiar se caracteriza por la interrelación entre propiedad, familia y negocio. Esta circunstancia de ser empresa familiar, presenta problemas frente al poder y la toma de decisiones. Al ser 
familiar, la "estructura societaria" dependerá no sólo del manejo del negocio sino también de su "arreglo burocrático", así como de la delegación de la autoridad en la gestión de la empresa (Vélez, Holguín, De la Hoz, Durán y Gutiérrez, 2008). En este tipo de empresas, los juegos de poder son más complejos dada la interrelación que posee, así como los poderes formales e informales, las situaciones de armonía y discordia, alianzas familiares, entre otros (De la Garza et al, 2008).

El control de la empresa es una gran preocupación del clan familiar, por tal razón, en la alta dirección se da la participación de los miembros de la familia, lo que puede conducir a mantener el control familiar, obtener independencia financiera, y subordinar los intereses empresariales a otros familiares (García y García, 2011).

La empresa familiar, también es utilizada por los miembros de la familia como un instrumento de poder (Galve y Salas, 2010). En la mayoría de las empresas familiares el control y el ejercicio del poder está en ocasiones "demasiado presente interceptando incluso o reconduciendo actuaciones" realizadas por los directivos de la sociedad, en el desarrollo de las actividades profesionales, lo cual provoca conflictos de "competencia profesional" (Giménez, 2002).

Uno de los problemas relacionados con el poder, que, además, es bastante crítico en la empresa familiar corresponde a los conflictos emocionales. Los intereses particulares de cada persona, grupo o coalición, dificulta el logro de la armonía y el desarrollo de los miembros de la familia en sus distintas dimensiones, para el logro de los objetivos de la empresa. Ello puede deberse a la crianza, el temperamento, la estructura organizacional, falta de una jerarquía clara, situaciones de estrés, clima poco agradable, así como también, las barraras de comunicación puesto que unos miembros tienen más po- der e influencia que otros (Lozano, 2003).

En otra perspectiva, Gimeno et al (2005), exponen que la empresa familiar tiene su origen en un fundador que crea la empresa según sus capacidades y necesidades. Con el trascurso de los años adquiere un valioso conocimiento, pero "el poder que el emprendedor tiene en la organización hace que no se vea obligado a abstraer y codificar dicho conocimiento para poderlo compartir con el resto del equipo directivo". La centralización en la toma de decisiones no hace necesario la disposición para todos del conocimiento, ni la necesidad de poner en marcha una estrategia deliberada; "la centralización permite la alineación de las decisiones sin la necesidad de compartir información relativa a la estrategia que cabe seguir" (Manzano y Ayala, 2006).

En lo relacionado con la dirección de la empresa, Van de Berghe y Levrau, (2004), consideran que es necesario generar un contrapeso al poder de los máximos ejecutivos en los consejos de administración, con el fin de garantizar una diversidad de conocimientos y experiencias, así como el logro de un equilibrio entre los distintos grupos de interés o coaliciones (Cabrera, Denis y Martín, 2011).

El poder en la empresa familiar también se analiza en la literatura desde diferentes ángulos de interés. En los trabajos académicos de responsabilidad social y empresa familiar, se asocia a este tipo de empresas con ciertos comportamientos que limitan el comportamiento responsable como el nepotismo, la ocultación de información, y la resistencia de las generaciones mayores a ceder el poder, tal como establecen los autores Gallo y Melé (1998), Lansberg (1988), Morck y Yeung (2003), Neubauer y Lank (1998), (Cabrera, et al, 2011). 
UNA REVISIÓN AL EJERCICIO DEL PODER Y EL CAMBIO ORGANIZACIONAL EN LA EMPRESA FAMILIAR

EL PODER, LA SUCESIÓN Y EL CAMBIO GENERACIONAL EN LA EMPRESA FAMILIAR

Uno de los momentos de cambio más críticos para estas empresas es la sucesión y el relevo generacional. De acuerdo con Lozano (2000), una sucesión inadecuada se constituye en uno de los grandes problemas de la empresa familiar.

Ginebra (2001), establece las siguientes generaciones: $1^{\mathrm{a}}$ generación: padre sólo más hijos; $2^{\mathrm{a}}$ generación: hermanos más primos; $3^{\mathrm{a}}$ generación: primos; $4^{\mathrm{a}}$ generación: primos segundos. El autor señala que el cambio intergeneracional puede romper los estrechos vínculos familiares desarrollados en la etapa inicial, por ello, la empresa debe cambiar su estructura cuando se pasa de una generación a otra para seguir existiendo. El conflicto intergeneracional aparece cuando se cambia la configuración de la empresa entre la primera y segunda generación. La reestructuración de poder se da entre ellas, así como en los niveles gerenciales (Navarro, Ramírez, Sánchez y Vaca, 2008).

Por lo general, en la primera generación, el modelo cultural dominante es el paternalista, caracterizado por relaciones jerárquicas donde el fundador $\mathrm{u}$ otros integrantes de la familia, mantienen para sí todo el poder en la toma de decisiones y la información de las operaciones más importantes de la empresa (Cabrera y Martin, 2010).

De acuerdo con Sheperd y Zahra (2003), algunas empresas familiares, a medida que corren los años muestran una aversión al cambio, siguiendo para ello, estrategias conservadoras que limitan el crecimiento y el éxito de la organización. Ciertos fundadores mantienen el control por un periodo de tiempo largo sin preparar líderes que sean capaces de asumir el cambio generacional. Como se expresó anteriormente, por lo general, el fundador se suele caracterizar por un poder muy fuerte que se prorro- ga en el tiempo, contrario a la repartición del poder (García y Sánchez, 2009).

Como es natural, en algún momento de la historia de la empresa familiar, se produce una atomización de la propiedad, la cual recaerá en una persona o grupo de personas que tienen relaciones de consanguinidad, el fundador -dirigente que lleva toda la carga del negocio, debe ceder con el paso de los años el poder a sus descendientes, pero la sucesión se retrasa en muchos casos porque no se sabe abordar el problema, siendo la resistencia o negación al retiro la posición más cómoda (Macías y Ramírez, 2011).

Hacia el momento de cambio generacional, el fundador o el líder presenta el natural temor a la muerte, la pérdida de poder y la actividad laboral después de décadas de estar en la cima; el sentirse ya inútil, hace que el fundador tenga una resistencia frente al retiro y se oponga a la sucesión, junto a otras razones relacionadas que dificultan el proceso (Jiménez, 2009).

La predisposición de un líder de irse o no irse también depende generalmente del miedo a perder la posición en la familia y a la comunidad donde reside. Si la tendencia del líder a abandonar el poder es cierta y visible, la probabilidad de una sucesión exitosa aumenta, puesto que ello indica la aceptación del líder de que es necesaria la sucesión, así como la aceptación de que su sucesor será un buen remplazo. Para el líder es importante la presencia de un sucesor comprometido con la empresa y con sus intereses (Manzano y Ayala, 2002).

En algunos casos, el fundador no se retira verdaderamente. Una investigación muestra que la salida de un fundador representa una división de responsabilidades entre los sucesores, pero los fundadores con tendencia centralizadora de toma de decisiones, siguen teniendo un peso significativo en la empresa 
después de la sucesión (Novaes et al, 2010). Dicho de otra forma: el líder, cuyo estilo de liderazgo sea el velar por el negocio, tiene mejores oportunidades de sucesión que aquel "monarca" o "general" cuyo estilo es glorificar el poder del liderazgo (Solomon, Breunlin, Panattoni, Gustafson, Ransburg, Ryan,, Hammerman, y Terrien, J. (2011).

La crisis de la segunda generación se relaciona con el dominio y la legitimación del poder. En la mezcla familia -empresa, la sucesión puede generar tensiones familiares que pueden llevar a la organización a un caos o producir nuevas relaciones entre los familiares y llevar a la organización por buen camino. Ello implica una planificación previa, hacer de la empresa un producto "apetecible" para los herederos, control en la transición, dejación del poder por parte del fundador o predecesor (Rodríguez, 2010:67,77). Lansberg y Astrachan (1994) establecen que la relación familiar juega un papel preponderante en la planeación del sucesor (Lozano y Urbano, 2010).

Un estudio realizado en México revela que las generaciones no se suceden rápidamente. La empresa familiar sobrepasa los 31 años, estando el $51 \%$ en manos de la segunda generación, lo que demuestra la aversión de los líderes de abandonar la gestión para las nuevas generaciones (Díaz y Fuentes, 2014).

En el trascurso del tiempo, las empresas familiares pueden tener problemas de legitimidad asociados a la sucesión del negocio. Al establecerse la sucesión, puede darse una falta de reconocimiento de quienes acceden al control de la actividad empresarial, independientemente de la legalidad con la que se hizo la sucesión; la experiencia demuestra que cuando existe confianza tiende a presentarse una disciplina muy flexible, donde la informalidad es vista como confianza lo cual rompe con él debe ser de la formalidad del negocio (Andrade, 2002)
La legitimidad y el liderazgo pueden ser dos aspectos muy complicados en la empresa familiar. El liderazgo tiende a ser confuso, puesto que, la sucesión de poder, muchas veces no se hace con el nombramiento de un líder; por ello, se considera que cada generación debe resolver sus propios conflictos y debe facultar y legitimar a sus descendientes (Goyzueta, 2013).

En definitiva, la relación entre padres e hijos, es importante para la colaboración y el buen funcionamiento de la empresa. Grabinsky (1992), expone que sí esta relación está enmarcada en términos de autoridad y poder, el choque y la confrontación hacen parte del día a día. Por un lado, el deseo del padre por afirmar su autoridad sobre los demás miembros, y, por el otro lado, el deseo de los hijos de adquirir poder. Esto tendrá repercusiones sobre la sucesión, y la tensión será fuerte o débil dependiendo de las características del núcleo familiar. Esto puede superarse con una relación cordial y el respeto (Albuquerque, Lozano y Ramírez, 2009).

Los estudios de situaciones relacionadas con sucesiones, sugieren un impacto negativo de los herederos en la empresa familiar (González, Guzmán, Pombo, Trujillo, 2011). Una hipótesis acerca de la decadencia de los grupos familiares en el tiempo, se debe a la dilución de la propiedad y del control por parte de los descendientes del fundador, también poderosos, que crean una carrera hacia el fracaso extrayendo las riquezas hacia afuera de la empresa (González et al, 2011)

El deseo de la continuidad familiar en la empresa también genera un orden de preferencias en cuanto a la financiación en función del riesgo de la pérdida de control de las distintas opciones; en este sentido se prefiere la financiación interna y si ésta no es suficiente se recurre a financiación externa, claro está, que a medida que pasan las generaciones disminuye la cuota de sacrificio y por lo tanto la 
posibilidad de autofinanciación (Blanco, De Quevedo y Delgado, 2009).

En las investigaciones de empresas familiares insanas o "adictivas", se muestra que mucha de la retórica de la empresa familiar, asume que el deseo de los hijos por pertenecer a la empresa y liderar sus procesos es algo bueno; pero las familias sufren fuerzas internas y externas que interfieren en el éxito. El "mantra" de la industria de la empresa familiar es: "sólo un tercio de las empresas familiares sobreviven a la segunda generación; al menos dos tercios fracasan en este traspaso generacional y cinco de cada seis empresas fracasan en el traspaso a la tercera generación" (Kaye, 1996:1). En la sociedad familiar se identifica una inestabilidad para las generaciones futuras debido a la desunión familiar, la no preparación de los sucesores por parte de los fundadores por falta de tiempo; en algunos casos, los fundadores se lamentan por haber sido exitosos en sus empresas, pero no en la familia (Gaona, De la Garza y Hernández, 2014).

Según Gallo (2002), el proceso de sucesión puede ser el principal problema para la empresa familiar, ya que muchas de ellas fracasan en su objetivo de crecimiento y continuidad. Pero debajo de la sucesión se suele esconder problemas como la maduración del mercado, la reestructuración de los miembros, conflictos familiares, el envejecimiento de la organización, entre otros, aspectos que deben tratarse en búsqueda de la solución sin interferir en el cambio generacional (Araya, 2012)

Para un proceso con mayores posibilidades de sucesión y relevo generacional se sugiere la utilización de herramientas como la promoción y el coaching. En el cambio de fundador a sucesor se recomienda el mentoring, para el desarrollo de las competencias requeridas (Núñez, Grande y Pedrosa, 2012). Una herramienta importante para garantizar un exitoso relevo generacional es el protocolo fami- liar (Martínez, 2013), considerado el elemento que más contribuye a la mejora de la empresa (Díaz y Fuentes, 2013), así como el instrumento más utilizado para "regularizar las cuestiones sucesorias" (De la Torre y Juanes, 2002). Gallo, también recomienda la práctica de los siguientes principios para un buen gobierno en la empresa familiar: el poder como servicio, transmisión responsable de la propiedad, desarrollar una comunidad de personas, una familia unida y comprometida (González, 2010).

\section{CONCLUSIONES}

1 poder pasó de ser un concepto represivo al
a concepto de las relaciones de poder. En el campo organizacional se le observa como influencia.

En los procesos de cambio organizacional, el poder toma fuerza, dado los movimientos que genera en el interior de la organización, en donde, posiblemente, unos tendrán más poder, $\mathrm{y}$, otros lo verán disminuir. Los cambios, dan lugar a juegos de poder en los que los grupos o coaliciones pretenden satisfacer sus intereses.

En la empresa familiar, el poder se particulariza dada sus características -propiedad, familia, negocio-. Los juegos de poder son muy complejos por la estructura presente. Incluso la empresa es utilizada como un instrumento de poder.

Aspecto crucial en la empresa familiar es el momento de la sucesión y el relevo generacional, en el cual el poder tiende a ser un obstáculo para darle paso a la siguiente generación. En la segunda generación se da una crisis de legitimización del poder. El éxito o no del cambio generacional, dependerá del modo en cómo se aborde el ejercicio del poder. Para un relevo exitoso los autores recomiendan, entre otras técnicas, el uso del protocolo familiar, y 
UNA REVISIÓN AL EJERCICIO DEL PODER Y EL CAMBIO ORGANIZACIONAL EN LA EMPRESA FAMILIAR

\section{REFERENCIAS BIBLIOGRÁFICAS}

Gibson, J., Ivancevich, J., Donnelly, J., Konopaske, R. (2006). Organizaciones. Comportamiento, estructura, procesos. Mexico: McGraw Hill

Morgan, Gareth (1998). Imágenes de la Organización. Alfaomega

Stoner, J., Freeman, R., Gilbert, D. (1996). Mexico: Administración. Prentice Hall

\section{REFERENCIAS ELECTRÓNICAS}

Acosta, C. (2002). Cuatro preguntas para iniciarse en cambio organizacional. Revista Colombiana de psicología, 11, 9-24. Recuperado de http:// www.revistas.unal.edu.co/index.php/psicologia/ article/view/1194

Andrade, J. (2002). Sucesión en la empresa familiar: su futuro cuando la muerte se acerca. Revista Venezolana de Gerencia, 7, (19), 375-389. Recuperado de http://www.redalyc.org/articulo.oa? $\mathrm{id}=29001903$

Albuquerque, Ayuzabet; Lozano, Oscar; Ramírez, Juan (2009). Organización, empresa y familia: de la empresa familiar a la organización familiar. Gestión y Estrategia, 36, 17-36. Recuperado de http://administracion.azc.uam.mx/descargas/ revistagye/rv36/rev36art01.pdf

Araya, A. (2012). El proceso de sucesión en la empresa familiar y su impacto en la organización. Tec Empresarial, 6, (2), 29-39. Recuperado de http://dialnet.unirioja.es/descarga/

articulo/4004912.pdf

Arras, A., Jáquez, J., Fierro, L. (2008). Comunicación y cambio organizacional. Revista Latina de Comunicación Social, 63, 418-434. Recuperado de http://

www.revistalatinacs.org/08/35_792_51_Chihuahua/
Ana_Maria_Arras.html

Ayala, J., Manzano, G. (2006). ¿La empresa familiar punto de partida o punto final en la aventura del emprendedor? Boletín de Estudios Económicos, 61 (181), 451-469. Recuperado de http:// search.proquest.com/docview/1348763359/

fulltextPDF/595D5EA86E82425BPQ/36? accountid $=49118$

Blanco, V., De Quevedo, E., Delgado, J.(2009). La estructura financiera de la empresa y el cambio generacional. Revista española de financiación y contabilidad, 38 (141), 57-73. Recuperado de http:// search.proquest.com/business/docview/920394192/ fulltextPDF/237A7DEBFBB148A1PQ/4?

accountid $=49118$

Bolaños, S., Pantoja, M., Jiménez, Á. (2009). Emprendimientos locales: El caso de las empresas familiares en el Sur Occidente colombiano. Tec Empresarial, 3, (3), 27-37. Recuperado de http:// dialnet.unirioja.es/descarga/articulo/3202474.pdf

Cabrera, K., Déniz, M., Martín, J. (2011). Consejos de administración y potencial para el desarrollo de la Responsabilidad Social en las empresas familiares no cotizadas españolas. Investigaciones Europeas de Dirección y Economía de la Empresa, 17 (3). Recuperado de http://search.proquest.com/ business/docview/1323957762/

fulltextPDF/237A7DEBFBB148A1PQ/151? accountid $=49118$

Cabrera, M., Martín, J. (2010). La influencia de las relaciones intergeneracionales en la formación y compromiso del sucesor: efectos. Revista Europea de Dirección y Economía de la Empresa, 19 (2), 111-118. Recuperado de http://search.proquest.com/ business/docview/859874613/ fulltextPDF/34C5103D23A5448APQ/1? accountid $=49118$

Chaux, C. (2006). Una mirada a las relaciones de 
UNA REVISIÓN AL EJERCICIO DEL PODER Y EL CAMBIO ORGANIZACIONAL EN LA EMPRESA FAMILIAR

poder en las organizaciones con una perspectiva compleja (Trabajo de grado de maestría). Universidad Nacional de Colombia, sede Manizales. Recuperado de http://www.bdigital.unal.edu.co/1229/1/ carmenelisachauxmayorga.pdf

De la Garza, M., Melchor, J., Jiménez, L. (2008). El poder en la empresa familiar. VI Congreso internacional de análisis organizacional. Nuevo Vallarta, Nayarit. Recuperado de http:// www.uacya.uan.edu.mx/VI_CIAO/

ponencias/7_familia/7_4.pdf

De la Torre, A., Juanes, R. (2002). La naturaleza de la empresa familiar y su continuidad. Boletín de Estudios Económicos, 57 (177), 451-466. Recuperado de http://search.proquest.com/business/ docview/1348762457/

fulltextPDF/2C30744E588142A6PQ/120?

accountid $=49118$

Díaz, H., Fuentes, E. (2013). La comunicación en la empresa familiar, principal elemento en la preservación de la armonía familiar. Revista Internacional Administración y Finanzas, 6, ( 3), 95-114. Recuperado de http://search.proquest.com/ docview/1459655317/

fulltextPDF/595D5EA86E82425BPQ/33?

accountid $=49118$

Díaz, H., Fuentes, E. (2014). Las reglas de convivencia y su incidencia en la continuidad de las empresas familiares de Tehuacan. Revista Internacional de Administración\& Finanzas, 7 (6), 63-73. Recuperado de ttp://search.proquest.com/business/ docview/1446439044/

fulltextPDF/237A7DEBFBB148A1PQ/172?

accountid $=49118$

Díaz, M. (2011). La empresa familiar y su organización en forma de sociedad mercantil, con especial referencia a la sociedad de responsabilidad limitada. Pevnia, 12, 1-70. Recuperado de http:// search.proquest.com/business/

docview/1288085705/fulltextPDF/

CAAF23D89E1432FPQ/15 accountid $=49118$

Egea, J. (2007). Protocolo familiar y pactos sucesorios. La proyectada reforma de los heredamientos. Indret, julio. Recuperado de http:// www.indret.com/pdf/455_es.pdf

Font, I., Gudiño, P., Sánchez, A. (2001). Poder y conflicto: aristas del cambio organizacional. Gestión y Estrategia, 20, 62-73. Recuperado de http:// administracion.azc.uam.mx/descargas/revistagye/ rv20/rev20pres.pdf

Galve, C., Salas, V. (2011). ¿Determinan la propiedad familiar diferencias en las relaciones entre preferencias, comportamientos y resultados en las empresa españolas cotizadas? Revista de Economía Aplicada, 19, (57), 5-34. Recuperado de http:// www.redalyc.org/articulo.oa?id=96922243001

Gaona, L. De la Garza, S., Hernández, R. (2014). El sucesor, figura clave en el éxito de la empresa familiar. Revista Internacional Administración \& Finanzas, 7 (3), 57-67. Recuperado de http://search.proquest.com/business/

docview/1445489192/

fulltextPDF/841D6F0931FC4432PQ/10?

accountid $=49118$

García, J., Sánchez, I. (2009). Amenazas y oportunidades. El reto de emprender la empresa familiar. Amenazas y oportunidades. Partida Doble, 212, 66-77. Recuperado de http:// revistacontable.dev.nuatt.es/noticias_base/el-reto-de -emprender-en-la-empresa-familiar-amenazas-yoportunidades

García, R., García, M. (2011). Estructura del consejo de administración en la empresa familiar versus no familia. Revista Española de Financiación y Contabilidad, 40 (149), 35-64. Recuperado de http://search.proquest.com/docview/920387909/ 
UNA REVISIÓN AL EJERCICIO DEL PODER Y EL CAMBIO ORGANIZACIONAL EN LA EMPRESA FAMILIAR

fulltextPDF/595D5EA86E82425BPQ/38?

accountid $=49118$

García, V. (2002). El cambio organizacional. Hitos de Ciencias Económico Administrativas, año 8, (21), 85-96. Recuperado de http:// www.ucapanama.org/ovasdo/

cambio_organizacional.pdf

Gimeno, A. (2004). El desempeño en la empresa familiar: un estudio causal de los factores y variables internas (Tesis de posgrado) Recuperado de www.biblioferrersalat.com/media/.../TESIS $\% 20 \mathrm{~A} \%$ 20GIMENO.pdf

Giménez, J. (2002). Riesgos y eficiencia de la empresa familiar. Boletín de Estudios Económicos, 57 (177), 395-432. Recuperado de http:// search.proquest.com/business/

docview/1348763611/

fulltextPDF/2C30744E588142A6PQ/127?

accountid $=49118$

González, M., Guzmán, A., Pombo, C.,; Trujillo, M. (2011). Revisión de la literatura de empresas familiares. Una perspectiva financiera. Academia, revista latinoamericana de administración, 47, 1842. Recuperado de http://www.redalyc.org/ articulo.oa? $\mathrm{id}=71618917003$

González, R. (2010). La continuidad de la empresa familiar. Anuario Jurídico y Económico Escurialense, 43, 401-410. Recuperado de http:// search.proquest.com/business/docview/864728324/ fulltextPDF/2C30744E588142A6PQ/109? accountid $=49118$

Goyzueta, S. (2013). Modelo de gestión para las empresas familiares con perspectivas de crecimiento y sostenibilidad. Perspectivas, año 6 (31), 87132. Recuperado de http://www.scielo.org.bo/ scielo.php? script $=$ sci_pdf\&pid $=$ S1994$37332013000100003 \& \operatorname{lng}=\mathrm{es} \& \mathrm{nrm}=\mathrm{iso} \& \mathrm{t} \operatorname{lng}=\mathrm{es}$
Jiménez, G. (2009). La empresa familiar. Su importancia y vigencia. IEFAC. 137p. Recuperado de http://www.ceipa.edu.co/sitio/sites/default/files/ images/Copia\%20de\%20Descargar\%20LIbro1.pdf

Kaye, K. (2005). Cuando la empresa familiar es una enfermedad. Traducción de Macías, Javier. En: Family Business Review, 1996, 9, 347-368, (Reprinted in The Dynamics of Family Business.) Editorial: iUniverse, Inc. Recuperado de http:// ceajournal.metro.inter.edu/spring10/

rodriguezcastillo0601.pdf

López, M., Restrepo, L., López, G. (2013). Resistencia al cambio en las organizaciones modernas. Scientia et Technica, 18 (1), 149 - 157. Recuperado de https://dialnet.unirioja.es/descarga/ articulo/4269616.pdf

Lozano, M. (2003). Las relaciones intrafamiliares en la empresa familiar. Pensamiento \& Gestión, 15, 83-110. Recuperado de http:// ciruelo.uninorte.edu.co/pdf/ pensamiento_gestion $/ 15 / 5$ _LAS $\%$ 20RELACIONES\%20INTRAFAMILIARES\% 20EN\%20LA\%20EMPRESA\% 20FAMILIAR_PENSAMIE.pdf

Lozano, M. (2000). El protocolo en las empresas de propiedad familiar. Estudios Gerenciales, vol 16, 74, 49-67. Recuperado de http:// bibliotecadigital.icesi.edu.co/biblioteca_digital/ bitstream/item/235/1/mlozano_proto-empfamiliar.pdf

Lozano, M., Urbano D. (2010). Pertenencia inicial de descendientes la empresa familiar. Revista venezolana de gerencia, año 15, (50), 183-206. Recuperado de http://www.scielo.org.ve/scielo.php? script $=$ sci_pdf\&pid $=$ S131599842010000200002\&lng=es\&nrm=iso\&tlng=es

Macías, V., Ramírez, D. (2011). Sucesión en empresas familiares. Análisis desde la teoría de la 
agencia. Caso: Caldas. Criterio Libre, 9 (15), 193212. Recuperado de http://search.proquest.com/ business/docview/1010350538/fulltextPDF/ CC4F89BBFBFD40E7PQ/18? accountid=49118

Martínez, N. (2013). La planificación sucesoria en las empresas familiares. Aspectos jurídicos (Trabajo de grado). Universidad Politécnica de Cartagena. Recuperado de http://repositorio.bib.upct.es/ dspace/bitstream/10317/3609/1/tfm310.pdf

Montealegre, J., Calderón, G. (2007). Relaciones entre actitud hacia el cambio y cultura organizacional. Estudio de caso en medianas y grandes empresas de confecciones en Ibagué. Innovar, 17 (29), 49-70. Recuperado de http://www.redalyc.org/ articulo.oa?id $=81802904$

Navarro, A., Ramírez, J., Sánchez, J., Vaca, G. (2008). La empresa familiar y el conflicto intergeneracional. Auge 21 (1). Recuperado de http:// www.auge21.net/documentos/articulos/ economia.administracion/la-empresa-familiar.pdf

Novaes, S., Maccari, E., Carneiro, J., Jordan, J. (2010). Sucessao em empresas familiares e seus impactos na estrategia empresarial: estudio de caso em empresasdo setor de fabricantes de produtos médicos. Revista iberoamericana de estrategia, 9 (3), 8811. Recuperado de http://search.proquest.com/ business/

docview/900652517/36BA00054E6E4C94PQ/9? accountid $=49118$

Núñez, P., Grande, F., Pedrosa, C. (2012). El reto de la dirección de los recursos humanos para las empresas familiares: estado de la cuestión. Revista Negotium, 23, 139-162. Recuperado de http:// www.revistanegotium.org.ve/

Osorio, L., Ravelo, E. (2011). Percepción del impacto del cambio organizacional en trabajadores de una institución universitaria de Bogotá: Una aproximación hermenéutica. Psychologia. Avances de la disciplina, 5 (1), 81-94 . Recuperado de http:// www.redalyc.org/pdf/2972/297224114008.pdf

Rodríguez, A. (1992). Poder y política en las organizaciones. Psicología Política, 5, 99-115. Recuperado de http://www.uv.es/garzon/psicologia\% 20politica/N5-5.pdf

Rodríguez, A. (2000). El poder. Una circunstancia en el pensamiento de Foucault. Revista de Ciencias Humanas, 24. Recuperado de http:// www.utp.edu.co/ chumanas/revistas/revistas/rev24/ rodriguez.htm

Rodríguez, L. (2010). Empresas familiares, ante la crónica de una muerte anunciada. En: Revista Empresarial Inter Metro, primavera, 6 (1), 62-88. Recuperado de http://ceajournal.metro.inter.edu/ spring10/rodriguezcastillo0601.pdf

Rodríguez, P., Pico, B., Méndez, F. (2013). Capacidad innovadora en la empresa familiar como área de oportunidad hacia el desarrollo de México. Economía, sociedad y territorio, 13 (43), 779-794. Recuperado de http://search.proquest.com/ docview/1448806078/

fulltextPDF/595D5EA86E82425BPQ/42? accountid $=49118$

Sanabria, R., Trujillo, M., Guzmán, A. (2008). Poder y estrategia. Investigación y Reflexión, 16 (2), 11-30. Recuperado de www.redalyc.org/ pdf/909/90916202.pdf

Savarse, C. (2013), Arquitectura estratégica ¿cómo alinear la estrategia con la organización? Orbis, 25, 21-32. Recuperado de http:// www.revistaorbis.org.ve/

Solomon, A,. Breunlin, D., Panattoni, K., Gustafson, M., Ransburg, D., Ryan, C., Hammerman, T., Terrien, J. (2011). Dont lock me out: life-story interviews of family business owners facing succession. Family Process, 50 (2), 149-166. Recuperado 
UNA REVISIÓN AL EJERCICIO DEL PODER Y EL CAMBIO ORGANIZACIONAL EN LA EMPRESA FAMILIAR

de http://search.proquest.com/socialsciences/ docview/870837796/

fulltextPDF/7421F4EDE2EB498DPQ/44? accountid $=49118$

Tapies, J. (2011). Empresa familiar: un enfoque multidisciplinar. Universia Businees Review, cuarto trimestre. Recuperado de http:// search.proquest.com/business/docview/964026666/ fulltextPDF/2C30744E588142A6PQ/108?

accountid $=49118$

Vallejo, M.C. (2005). Cuando definir es una necesidad. Una propuesta integradora y operativa del concepto de empresa familiar. Investigaciones Europeas de Dirección y Economía de la Empresa, 11 (3), 151-171. Recuperado de http:// dialnet.unirioja.es/descarga/articulo/1357983.pdf

Vélez, D., Holguin, H., De la Hoz, H., Durán, Y., Gutiérrez, I. (2008). Dinámica de la empresa familiar PYME. Estudio exploratorio en Colombia. Fundes. Recuperado de http://www.google.com.co/ url?

$\mathrm{sa}=\mathrm{t} \& \mathrm{rct}=\mathrm{j} \& \mathrm{q}=\&$ esrc $=\mathrm{s} \&$ source $=$ web $\& \mathrm{~cd}=10 \& \mathrm{ved}$ $=0 \mathrm{CGUQFjAJ} \& u r l=h t t p \% 3 \mathrm{~A} \% 2 \mathrm{~F} \%$

2Fwww.mundopymeabm.org.mx $\% 2$ Fpdf $\%$

2FDinamicaEmpresaFamiliarFUN-

DES.pdf\&ei=mRANU6quOqfMsATLvoCgCA\&us $\mathrm{g}=$ AFQjCNHW4ue7hK0BCvsrebDlOZcnIj5Oog\&b $\mathrm{vm}=$ bv.61725948,d.cWc

Zuluaga, P. (2010). Una perspectiva de las empresas de familia. El caso de las empresas de familia manizaleñas (Trabajo de posgrado). Universidad Nacional de Colombia sede Manizales. Recuperado de http://www.bdigital.unal.edu.co/1803/1/ paulazuluagaarango.2010.pdf

Manzano, G., Ayala, J. (2002). Sucesión en la empresa familiar: algunas claves del éxito. Boletín de Estudios Económicos, 57 (177), 433-449. Recuperado de http://search.proquest.com/business/ docview/1348762452/

fulltextPDF/2C30744E588142A6PQ/101?

accountid $=49118$ 